Check for updates

Cite this: RSC Adv., 2018, 8, 34162

Received 22nd June 2018

Accepted 21st September 2018

DOI: $10.1039 / c 8 r a 05368 \mathrm{e}$

rsc.li/rsc-advances

\section{Phosphorescent iridium-containing nanomicelles: synthesis, characterization and preliminary applications in nanomedical imaging $\dagger$}

\author{
I. Monaco, ${ }^{a}$ M. Maturi, ${ }^{a}$ E. Matteucci, (D) ${ }^{a}$ E. Locatelli, (D) ${ }^{a}$ A. Baschieri, (D) ${ }^{a}$ P. Zani, ${ }^{a}$ \\ P. Armanetti, ${ }^{\text {b }}$ L. Menichetti, ${ }^{\text {b }}$ L. Sambri iD *a and M. Comes Franchini iD *a
}

\begin{abstract}
Diagnostic nanomedicine constantly requires the development of novel contrast agents with intrinsic imaging capabilities. Phosphorescent Ir(III)-complexes represent good candidates when delivered through polymeric nanoparticles. In this work, we propose a biocompatible nanoparticle made from an intrinsically phosphorescent copolymer, synthesized directly with an imaging tag present on its backbone. Polymeric nanoparticles can be obtained with the exact amount of phosphorescent moieties needed to maximize their output signal. Complete characterization and ex vivo studies confirmed that this nanosystem is suitable as a future diagnostic tool.
\end{abstract}

\section{Introduction}

Nanoparticle technology has recently been shown to hold great promise for drug delivery applications in nanomedicine due to its beneficial properties such as better encapsulation, bioavailability, controlled release, and a lower toxic effect. These concepts may even be of high impact when in conjugation with an imaging/diagnostic approach. Indeed, due to a fast advance of imaging techniques, we now have the opportunity to map biochemical pathways in vivo at an unprecedented level., ${ }^{\mathbf{1 , 2}}$

There are many ways to perform imaging, which are widely reviewed and commonly used in synthetic laboratories, ranging from metal/metal oxides to fluorescent/phosphorescent diagnostic agents. ${ }^{3}$ Among them, optical imaging has emerged as an easy, non-ionizing and flexible imaging modality, widely used in experimental studies to non-invasively differentiate between lesions and healthy tissues on the basis of differential dye extraction.

Recently, the use of phosphorescent transition metal complexes, such as cyclometalated Ir(III)-complexes, as intracellular sensors and bioimaging reagents has received great attention. ${ }^{4,5}$ This is by virtue of some of their unique properties, such as large Stokes' shifts, high quantum yields, long-lived phosphorescence, high photostability, and cell permeability, making them ideal candidates for bioimaging agents.

From a synthetic point of view, a straightforward approach is to conjugate the "diagnostic tag" onto the surface of the nanosystems used for drug delivery. The chemistry is simple and well

${ }^{a}$ Department of Industrial Chemistry "Toso Montanari", Viale Risorgimento 4, Bologna, Italy. E-mail: mauro.comesfranchini@unibo.it

${ }^{b}$ CNR Institute of Clinical Physiology, Via Moruzzi, Pisa 1 56124, Italy

$\dagger$ Electronic supplementary information (ESI) available: Synthesis of ligand 12. See DOI: 10.1039/c8ra05368e understood; a major concern is how to carefully tune the functional groups on both reactive partners. However, some minor drawbacks exist using this approach: (i) after the conjugation steps, a new full characterization of the final nanosystem is needed (the size, zeta-potential, percentage of entrapped drug and percentage of the attached diagnostic tag); (ii) it is not really known how something on the surface of the nanosystem might affect interactions with the other parts in vitro and/or in vivo; (iii) the fate of the diagnostic tag is something that is rarely considered, and its toxicity $v s$. active percentage has to be carefully determined once attached; and (iv) the ligand is commonly accepted from a chemical point of view, but destructive interactions in vivo cannot be readily ruled out.

An alternative and useful approach would be to have a robust nanosystem containing a strong diagnostic phosphorescent tag chemically linked to the polymer. In this case the polymer precursor of the nanosystem could be stored containing the tag and used to prepare the imaging nanosystem. No extra conjugation step would be required and the fate of the "organic matter" could be easily tracked due to its linkage with the tag.

A search through the literature has shown, surprisingly, very few reports based on this approach.

Difluoroboron dibenzoylmethane-polylactide as nanoparticles has been also reported showing unique emission properties in an aqueous environment. ${ }^{6}$ Magneto-phosphorescent d-f coordination polymer nanoparticles were synthesized from phosphorescent carboxyl-functionalized iridium complexes and magnetic Gd(III) ions. $^{7}$ Polyfluorene or poly(fluorene-alt-benzothiadiazole) was covalently bound to a phosphorescent metalloporphyrin (PtTFPP, PtTPTBPF) and a class of nanoparticles were prepared by a precipitation method. ${ }^{8}$

Fluorescent organic nanoparticles (FONs) based on aggregation induced emission (AIE) dyes were obtained in a one-pot 
strategy by using a hydrophobic AIE dye with a hydroxyl end functional group and a hydrophilic polyethylene glycol monomethyl ether attached onto the methacrylate monomer via enzymatic transesterification. RAFT polymerization of the functionalized methacrylate monomers was then performed, which self-assembled into FONs with the hydrophobic AIE core covered by a hydrophilic PEG shell. ${ }^{9}$ In a similar approach, fluorescent polymeric nanoparticles (FPNs) were obtained via an anhydride ring-opening polycondensation and subsequent cross-linking with an AIE monomer with two amino endgroups. ${ }^{10}$

To the best of our knowledge, the most recent manuscript was by Huang, who reported semiconducting polymer dots (Pdots) composed of phosphorescent Ir(III) complexes and polyfluorene units. ${ }^{11}$

It seems clear that there is very limited research on developing such kinds of phosphorescent polymer for applications in sensing and bioimaging.

We therefore propose a straightforward chemical synthesis to incorporate a cyclometalated Ir(III) complex ${ }^{12,13}$ with its longlived phosphorescence into a well-known, ${ }^{14}$ non-toxic ${ }^{15}$ biodegradable and biocompatible polymer classified as GRAS (Generally Recognized As Safe): poly lactic-co-glycolic acid (PLGA). ${ }^{16}$

\section{Experimental section}

\section{Materials and methods}

Reagents were purchased from Sigma Aldrich and used as received unless stated otherwise. Dynamic light scattering (DLS) measurements were performed on a Malvern Zetasizer nano-S equipped with a $532 \mathrm{~nm}$ laser beam. $\zeta$-Potential measurements were conducted in DTS1060C-Clear disposable zeta cells at $25{ }^{\circ} \mathrm{C}$. Chromatographic purifications were performed using silica gel. ${ }^{1} \mathrm{H}$ and ${ }^{13} \mathrm{C}$ NMR spectra were recorded on Varian Inova (300 and $600 \mathrm{MHz}$ for ${ }^{1} \mathrm{H}$ ) and Varian Mercury $(400 \mathrm{MHz}$ for ${ }^{1} \mathrm{H}$ ) spectrometers. Chemical shifts $(\delta)$ are reported in ppm relative to the residual solvent signals for ${ }^{1} \mathrm{H}$ and ${ }^{13} \mathrm{C} \mathrm{NMR}\left({ }^{1} \mathrm{H}\right.$ NMR: 7.26 ppm for $\mathrm{CDCl}_{3} ;{ }^{13} \mathrm{C}$ NMR: $77.0 \mathrm{ppm}$ for $\left.\mathrm{CDCl}_{3}\right) \cdot{ }^{13} \mathrm{C}$ NMR spectra were acquired using a ${ }^{1} \mathrm{H}$ broad band decoupled mode. Coupling constants are given in $\mathrm{Hz}$. The absorption properties of the PLGA-12/TPGS@PNPs-Ir were investigated with an UV-Vis spectrometer (Perkin Elmer, Inc.) equipped with an integrating sphere. Steady-state emission spectra were recorded on an Edinburgh FLS920P spectrofluorimeter equipped with a $450 \mathrm{~W}$ Xenon arc lamp, double excitation and single emission monochromators and a Peltier cooled Hamamatsu R928P photomultiplier tube (185-850 nm). Concentration of the final solution was determined by drying a fixed amount of solution for two days at $110{ }^{\circ} \mathrm{C}$ and weighing the residual organic material. A Photonimager Rt by Spacelab was used to perform the phosphorescence tests in vitro and ex vivo.

\section{Synthesis of PLGA-12}

To a solution of PLGA-COOH (7 kDa, $350 \mathrm{mg}, 0.05 \mathrm{mmol})$ in anhydrous DCM (5 mL) was added $N$-hydroxysuccinimide (NHS,
$22 \mathrm{mg}, 0.188 \mathrm{mmol}$ ) and the solution was cooled to $0{ }^{\circ} \mathrm{C}$ before addition of $N, N^{\prime}$-dicyclohexylcarbodiimide (DCC, $42 \mathrm{mg}, 0.22$ $\mathrm{mmol}$ ). The mixture was left to react for 24 hours at room temperature then filtered on Celite®. The polymer (PLGANHS) was precipitated and washed twice with cold $\mathrm{Et}_{2} \mathrm{O}$ then dried in vacuo in order to obtain a white solid (quantitative yield, $355 \mathrm{mg}, 0.05 \mathrm{mmol}$ ) that must be stored at $-20{ }^{\circ} \mathrm{C}$ until usage.

The obtained PLGA-NHS was dissolved in $\mathrm{CHCl}_{3}(6 \mathrm{~mL})$ and $12(24 \mathrm{mg}, 0.05 \mathrm{mmol})$, obtained as reported in the ESI $\dagger$ and previously dissolved in $1 \mathrm{~mL}$ of $\mathrm{CHCl}_{3}$, was added. DIPEA ( $20 \mu \mathrm{L}, 0.1 \mathrm{mmol}$ ) was also added. The mixture was left to react for 24 hours, then the resulting PLGA-12 was precipitated and washed twice with cold $\mathrm{Et}_{2} \mathrm{O}$. This was then dried in vacuo in order to obtain a white solid $(310 \mathrm{mg}, 0.044 \mathrm{mmol}$, yield = $88.5 \%)$.

\section{Synthesis of PLGA-12-Ir}

PLGA-12 (352 mg, $0.05 \mathrm{mmol}$ ) was dissolved in a 3/1 DCM/EtOH mixture $(32 \mathrm{~mL})$ and $\left[\operatorname{Ir}(\text { ppy })_{2}(\mu-\mathrm{Cl})\right]_{2}(53.5 \mathrm{mg}, 0.05 \mathrm{mmol})$ was added. The solution was left to stir for $16 \mathrm{~h}$ at $\mathrm{rt}$. After solvent evaporation, the crude solid was dissolved in DCM and precipitated from $\mathrm{Et}_{2} \mathrm{O}$ three times. The obtained solid was washed with $\mathrm{Et}_{2} \mathrm{O}$, then dried in vacuo to obtain PLGA-12-Ir (337 mg, $0.046 \mathrm{mmol}, 92 \%$ yield) as a yellow solid.

\section{Synthesis of PLGA-12/TPGS@PNPs-Ir}

A solution of PLGA-12 (from 0 to $25 \mathrm{mg}$ ) and PLGA-12-Ir (from 25 to $0 \mathrm{mg}$ ) in $2.5 \mathrm{~mL}$ of chloroform was prepared. This organic mixture was sonicated ( $600 \mathrm{~W}$ input, $40 \%$ amplitude, $3 \mathrm{~min}$ ) with $25 \mathrm{~mL}$ of water containing $50 \mathrm{mg}$ of $\mathrm{D}-\alpha$-tocopherol polyethylene glycol 1000 succinate (TPGS), in an ice bath. The organic solvent was removed under vacuum, and the nanoparticles were purified and concentrated to $2 \mathrm{~mL}$ using centrifugal filter devices (Amicon Ultra, Ultracel membrane with 100.000 NMWL, Millipore).

\section{Phosphorescence measurements}

The PLGA-12/TPGS@PNPs-Ir obtained with a PLGA-12-Ir content of $50 \%$ were loaded in polyethylene (PE) tubes at different Ir concentrations: $15.2 \mu \mathrm{M}, 7.60 \mu \mathrm{M}, 3.80 \mu \mathrm{M}, 1.90 \mu \mathrm{M}$ and $0.76 \mu \mathrm{M}$. A PE tube filled with ultrapure milliQ water was used as the background. After loading, the PE tubes were arranged coplanarly inside the Photonimager RT. The wavelength of excitation ( $\left.\lambda_{\text {exc }}\right)$ was $400 \mathrm{~nm}$ and we checked the emission at $480 \mathrm{~nm}\left(\lambda_{\mathrm{em}}\right)$. We carried out four acquisitions at different times and acquired the respective phosphorescence images, in which we drew equal regions of interest (ROI), where we calculated the photon emission (PHsE) provided by PLGA12/TPGS@PNPs-Ir. After that, we performed phosphorescence tests in chicken breast tissue models. As reported in the literature, ${ }^{17,18}$ chicken breast offers a good tissue model for studying phosphorescence properties of potential contrast agents. Hence, we injected two samples of chicken breast by syringe with a bolus of around $100 \mu \mathrm{L}$, with one injection per sample. The injections were made respectively around 1 and $0.5 \mathrm{~mm}$ 


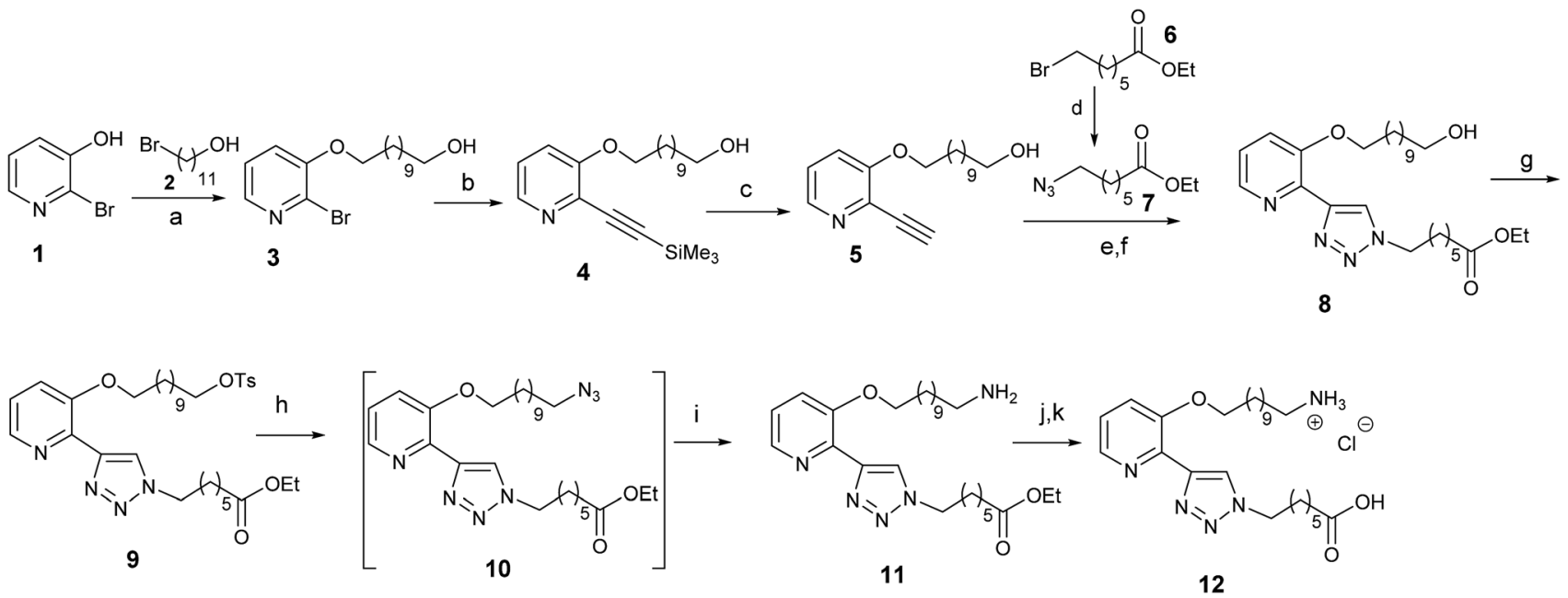

Scheme 1 Synthetic route to functionalized 4-pyridyl-1,2,3-triazole derivative 12. Reagents and conditions: (a) $\mathrm{K}_{2} \mathrm{CO}_{3}, 11-\mathrm{Br}-\mathrm{undecan}-1$-ol 2, acetone, reflux, $19 \mathrm{~h}$; (b) trimethylsilylacetylene, Cul, $\mathrm{Pd}\left(\mathrm{PPh}_{3}\right)_{2} \mathrm{Cl}_{2}, \mathrm{TEA}, \mathrm{THF}, \mathrm{rt}, 2 \mathrm{O}$; ( (c) TBAF, $\mathrm{THF}, \mathrm{H}_{2} \mathrm{O}, 0{ }^{\circ} \mathrm{C} \rightarrow \mathrm{rt}, 5 \mathrm{~h}$; (d) NaN3, DMSO, rt, 5 h; (e) $\mathrm{CuSO}_{4} \cdot 5 \mathrm{H}_{2} \mathrm{O}, \mathrm{Na}$-ascorbate, $t-\mathrm{BuOH} / \mathrm{H}_{2} \mathrm{O} 1$ : 1, rt, 24 h; (f) aq. $\mathrm{NH}_{3}, \mathrm{DCM}, \mathrm{rt}, 24 \mathrm{~h}$; (g) TsCl, pyridine, DCM, $0{ }^{\circ} \mathrm{C}, 24 \mathrm{~h}$; (h) NaN3, DMSO, rt, 5 h; (i) $\mathrm{PPh}_{3}, \mathrm{THF}, \mathrm{H}_{2} \mathrm{O}, 48 \mathrm{~h}$; (j) KOH, DCM/EtOH 2 : 1, rt; and (k) $2 \mathrm{M} \mathrm{HCl}$ in $\mathrm{Et}_{2} \mathrm{O}, \mathrm{DCM}$, rt.

deep for Sample 1 and Sample 2, maintaining the needle parallel to the chicken surface during the injection. Then, the injected ex vivo samples, with one more non-injected like the background, were placed and stimulated as described above. For both the test-objects and ex vivo samples, we evaluated the values of contrast, SNR and CNR.

\section{Results and discussion}

\section{Synthesis and characterization}

The synthesis of the linker $\mathbf{1 2}$ is reported in Scheme 1 . Commercial 2-bromo-3-hydroxy pyridine $\mathbf{1}$ was alkylated and subsequently reacted with ethynyl-trimethylsilane to give the bifunctional derivative 4. After removal of the trimethylsilyl group, a click reaction of 5 with azide 7 gave the functionalized bidentate ligand $\mathbf{8}$. The hydroxyl group in $\mathbf{8}$ was then transformed into an amino group, through a protection and nucleophilic substitution reaction sequence followed by reduction of the formed azide, to give 11. Finally, the desired ligand $\mathbf{1 2}$ was obtained through ester saponification followed by acidification.
Table 1 DLS and ICP-MS results obtained for the PLGA-12/ TPGS@PNPs-Ir

\begin{tabular}{lllll}
\hline $\begin{array}{l}\text { PLGA-12-Ir } \\
(\mathrm{w} / \mathrm{w} \%)\end{array}$ & $\begin{array}{l}\text { Iridium } \\
\left(\mu \mathrm{g} \mathrm{mL}^{-1}\right)\end{array}$ & $\begin{array}{l}\text { Diameter } \\
(\mathrm{nm})\end{array}$ & PDI & $\begin{array}{l}\zeta \text {-Potential } \\
(\mathrm{mV})\end{array}$ \\
\hline 1 & & & & \\
5 & 0.112 & $156.2 \pm 0.7$ & $0.216 \pm 0.1$ & -7.2 \\
10 & 0.181 & $164.3 \pm 0.5$ & $0.202 \pm 0.2$ & -6.9 \\
25 & 0.196 & $159.0 \pm 0.9$ & $0.215 \pm 0.1$ & -7.2 \\
50 & 0.221 & $179.6 \pm 1.6$ & $0.126 \pm 0.2$ & -8.3 \\
100 & 1.78 & $167.0 \pm 1.8$ & $0.160 \pm 0.1$ & -6.4 \\
& 3.17 & $158.3 \pm 1.9$ & $0.214 \pm 0.1$ & -7.8
\end{tabular}

Ligand 12 was anchored at one end of the biocompatible copolymer PLGA-COOH thanks to activation of the free carboxylic acid as the NHS ester and subsequent amide bond formation with the amino group of ligand 12, (Scheme 2). Complexation with Ir was carried out by stirring PLGA-12 with $\left[\operatorname{Ir}(\mathrm{ppy})_{2}(\mu-\mathrm{Cl})\right]_{2}$ (where Hppy $=2$-phenylpyridine) in DCM/ $\mathrm{EtOH}=3 / 1,16 \mathrm{~h}$ at room temperature, to produce the
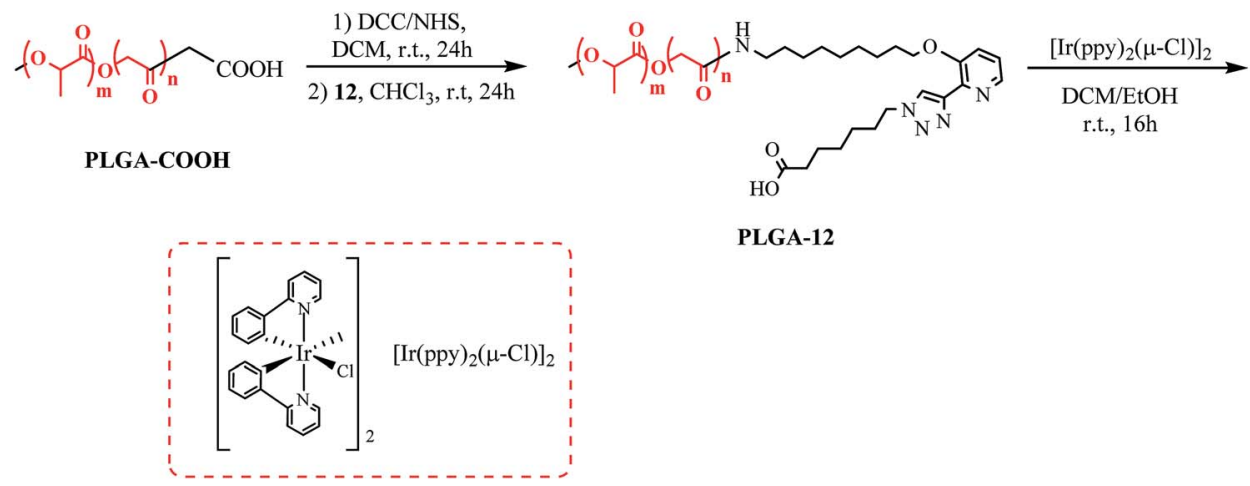

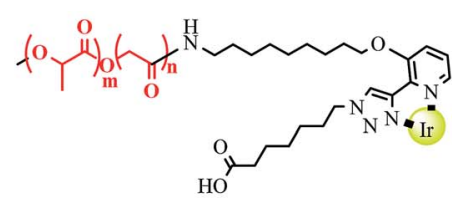

PLGA-12-Ir

Scheme 2 Procedure for the synthesis of PLGA-12-Ir. 


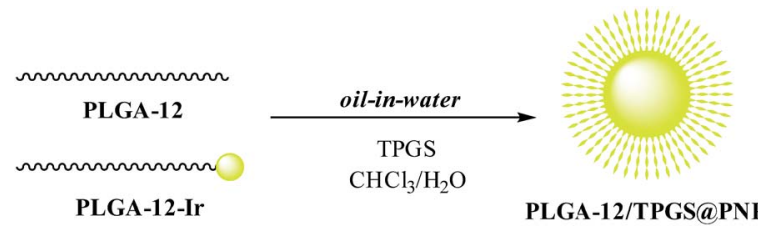

PLGA-12-Ir

PLGA-12/TPGS@PNPs-Ir

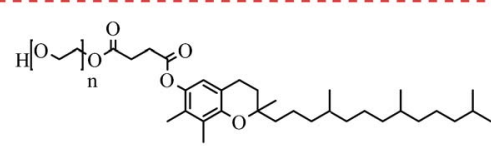

D-a-Tocopherol polyethylene glycol 1000 succinate Vitamin E PEG (TPGS)

Scheme 3 Schematic representation of the synthesis of PLGA-12/ TPGS@PNPs-Ir.

corresponding phosphorescent polymer PLGA-12-Ir in 92\% overall yield.

The synthesis of the PLGA-12/TPGS@PNPs-Ir was carried out through a single-emulsion solvent-evaporation technique. ${ }^{19}$ Briefly, PLGA-12 and PLGA-12-Ir were dissolved in $\mathrm{CHCl}_{3}$ at various blend ratios, in order to obtain nanomicelles characterized by different iridium percentages (Table 1). An aqueous solution was prepared by dissolving vitamin E PEG (TPGS) in water. The two solutions were mixed, forming a biphasic solution that was emulsified with a tip probe sonicator. The organic solvent present in the resulting suspension was removed
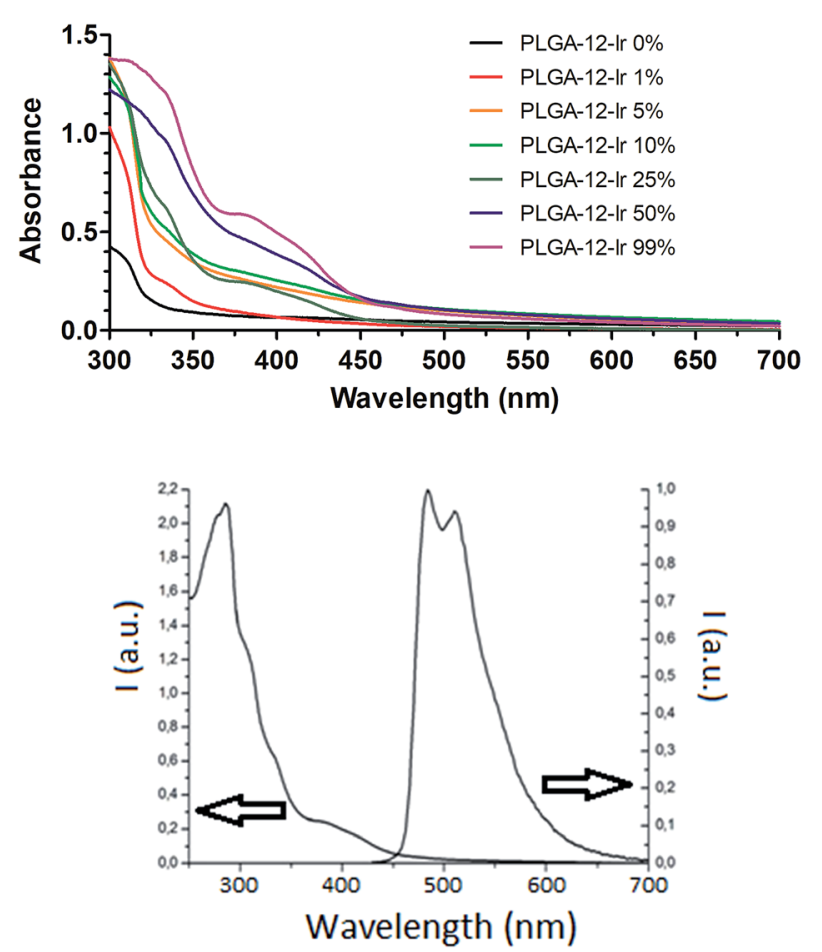

Fig. 1 (Top) UV-Vis absorption spectra obtained in water on PLGA-12/ TPGS@PNPs-Ir for different PLGA-12-Ir content. (Bottom) Excitation and emission spectra of PLGA-12/TPGSAPNPs-Ir 50\%.
Table 2 Phosphorescence data and the values of contrast, SNR and CNR obtained for the PLGA-12/TPGS@PNPs-Ir

\begin{tabular}{lllllll}
\hline Conc. $(\mu \mathrm{M})$ & \#PHsE average & St.-dev. & C.V.\% & Contrast & SNR & CNR \\
\hline 15.2 & $7.58 \times 10^{6}$ & $201 \times 10^{3}$ & 2.66 & 10.73 & 38 & 27 \\
7.6 & $3.92 \times 10^{6}$ & $156 \times 10^{3}$ & 4.00 & 5.07 & 25 & 15 \\
3.8 & $2.14 \times 10^{6}$ & $164 \times 10^{3}$ & 7.65 & 2.32 & 13 & 7 \\
1.9 & $1.48 \times 10^{6}$ & $145 \times 10^{3}$ & 9.84 & 1.28 & 10 & 4 \\
0.76 & 941837 & $107 \times 10^{3}$ & 11.39 & 0.46 & 9 & 1 \\
MilliQ & 646296 & $159 \times 10^{3}$ & 24.62 & & &
\end{tabular}

through rotary evaporation, until an opalescent brilliant yellow solution was obtained. The formed PLGA-12/TPGS@PNPs-Ir was purified in order to eliminate small unreacted molecules (Scheme 3)

The obtained PLGA-12/TPGS@PNPs-Ir were analysed with a Dynamic Light Scattering (DLS) technique in order to investigate the size and the polydispersity index of the nanomicelles. In addition, Inductively Coupled Plasma Mass Spectrometry (ICP-MS) analyses were performed to calculate the amount of iridium present in the samples. The DLS results (Table 1) revealed that the different percentages of metal do not affect the dimensions and zeta potentials of the nanomicelles, leading to the formation of systems characterized by a hydrodynamic diameter around $160 \mathrm{~nm}$ and a slight negative charge on the PNPs' surface. On the other hand, the ICP results showed that

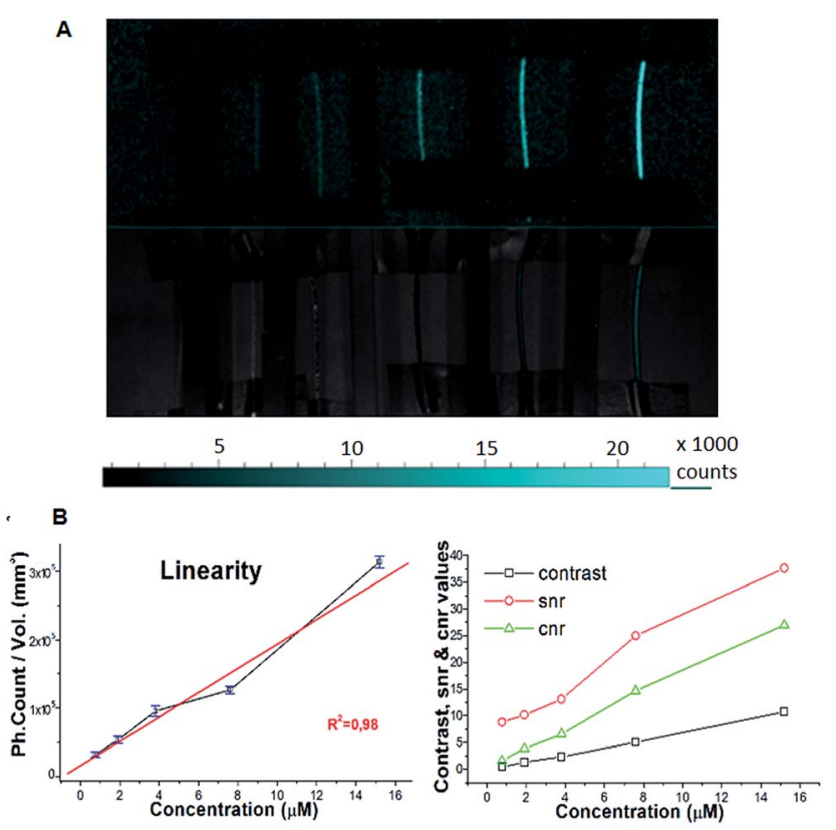

Fig. 2 (A) (top) Phosphorescence photon emission of PLGA-12/ TPGS@PNPs-Ir loaded in PE tubes at $15.2 \mu \mathrm{M}, 7.60 \mu \mathrm{M}, 3.80 \mu \mathrm{M}, 1.90$ $\mu \mathrm{M}$ and $0.76 \mu \mathrm{M}$ (from left to right). The last tube was filled with MilliQ water. (bottom) A processed phosphorescence image with a cyan scalebar for photon emission counting. (B) (left) The linear correlation between the PHsE and the concentration of PLGA-12/TPGS@PNPs-Ir loaded in PE; and (right) the trends in the image quality parameters with concentration. 

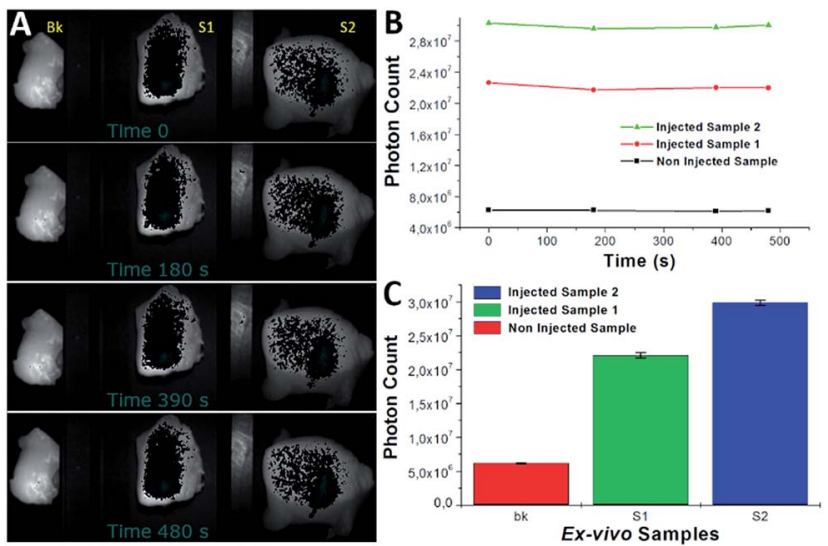

Fig. 3 (A) From left to right, three ex vivo samples of chicken breast: the first one was non-injected (Bk), while the second one (S1) and the last one (S2) were injected with a $100 \mu \mathrm{L}$ bolus of PLGA-12/ TPGS@PNPs-Ir at 1 and $0.5 \mathrm{~mm}$ in depth respectively. From top to bottom are the different times of acquisition. (B) The plot of photon emission (PHsE) acquired during the acquisition at different times. (C) Average of the PHsE count of the whole time provided from the chicken breast samples.

the amount of iridium in the nanomicelles was proportional to the amount of PLGA-12-Ir used in the synthesis.

The photophysical properties of the obtained nanoparticles in an air-equilibrated water solution were investigated. The absorption spectra of the PLGA-12/TPGS@PNPs-Ir containing increasing amounts of $\operatorname{Ir}(\mathrm{III})$, are reported in Fig. 1. The intense peak at $260-290 \mathrm{~nm}$ can be ascribed to the PLGA polymer chains, as confirmed by the PLGA-NPs spectrum used as a blank reference. Analogous to what we reported for other Ir(III)-containing nanoparticles and other similar complexes,${ }^{20}$ the weaker absorption bands in the $320-420 \mathrm{~nm}$ region belong to spin allowed metal-to-ligand charge-transfer ${ }^{1}$ MLCT and spin forbidden ${ }^{3}$ MLCT transitions of the Ir(III)-complex, and their intensities increase with increasing iridium concentration in the nanoparticles.

The stability of the nanoparticles was investigated by performing an analysis of the nanoparticle suspension in water, kept at $+4{ }^{\circ} \mathrm{C}$ for 2 months after preparation, and by comparing the obtained results with an analysis performed immediately after the synthesis. DLS analysis showed no variation in either the mean hydrodynamic diameter $(168.5 \pm 2.4 \mathrm{~nm})$ or in the $\zeta$ potential value $(-8.1 \mathrm{mV})$. Equally there was no significant variation in the emission profile (see Fig. S1†) showing that the particles can be considered stable in the reported conditions.
In addition, the emission properties of the obtained nanoparticles were measured: all of the batches displayed similar behavior, therefore we reported only the data for PLGA-12/ TPGS@PNPs-Ir obtained using 50\% of PLGA-12-Ir. The nanoparticles exhibit intense phosphorescence with emission maxima at 484 and $512 \mathrm{~nm}$ (Fig. 1), with an average quantum yield $(\Phi)$ of $24 \pm 5 \%$ and an average lifetime of $1.06 \pm 0.05 \mu \mathrm{s}$. These results indicate that the $\operatorname{Ir}(\mathrm{III})$ complex is able to maintain its phosphorescence properties even when directly linked to a polymer and once assembled into nanoparticles, making the novel obtained nano-objects fascinating candidates for bioimaging studies.

\section{Ex vivo studies}

Test-object experiments. As reported in Table 2, the PHsE was stable for each dilution with a percentage variation coefficient in the range of around 2 to $10 \%$. A linear correlation was found between the PHsE and the concentration (Fig. 2A and B) with $R^{2}=0.98$. The minimum effective concentration was estimated to be around $1.25 \mu \mathrm{M}$. The calculations of contrast, the CNR and SNR showed values increasing with concentration as reported in Table 2 and Fig. 2.

Ex vivo experiments. As shown in Fig. 3, after the injection the PLGA-12/TPGS@PNPs-Ir perfused the chicken breast tissue (Fig. 3A), providing a constant emission during all of the time measurements (Fig. 3B). The count of the PHsE from injected chicken breast samples was one order of magnitude higher (Fig. 3C).

All of the samples showed a stable PHsE with a standard deviation 3 orders of magnitude lower (Table 3). The calculations of contrast, SNR and CNR with respect to the non-injected sample gave high values, which made them easily discriminable inside the tissue.

\section{Conclusions}

In conclusion, we modified the biocompatible copolymer PLGA with a specific ligand able to cyclometalize Ir(III), thus obtaining an intrinsic phosphorescent polymer. Nanomicelles were then obtained from this phosphorescent copolymer with an oil-in-water emulsion technique and were widely characterized, demonstrating an efficient optical imaging contrast. This work highlights the potential of nanoscale phosphorescent iridium-containing micelles as a novel platform for the design of luminescent hybrid nanoparticles with high contrast for optical imaging and nanomedicine applications.

Table 3 Phosphorescence data from ex vivo samples: the mean values of number of photons emitted, standard deviation, coefficient of variation, contrast, SNR and CNR

\begin{tabular}{lccccc}
\hline Ex vivo sample & \#Photons average & St. dev. & C.V.\% & Contrast & SNR \\
\hline Sample 1 & $29.93 \times 10^{6}$ & $315 \times 10^{3}$ & 1.06 & 4 & 95 \\
Sample 2 & $22.07 \times 10^{6}$ & $390 \times 10^{3}$ & 1.77 & 3 & 54 \\
Bk & $6.12 \times 10^{6}$ & $62 \times 10^{3}$ & 1.01 & & 40
\end{tabular}




\section{Conflicts of interest}

There are no conflicts to declare.

\section{Notes and references}

1 T. Ramasamy, H. B. Ruttala, B. Gupta, B. K. Poudel, H. G. Choi, C. S. Yong and J. O. Kim, J. Controlled Release, 2017, 258, 226-253.

2 P. Padmanabhan, A. Kumar, S. Kumar, R. K. Chaudhary and B. Gulyás, Acta Biomater., 2016, 41, 1-16.

3 O. S. Wolfbeis, Chem. Soc. Rev., 2015, 44(14), 4743-4768.

4 K. K.-W. Lo and K. K.-S. Tso, Inorg. Chem. Front., 2015, 2(6), 510-524.

5 K. K.-W. Lo, Acc. Chem. Res., 2015, 48(12), 2985-2995.

6 A. Pfister, G. Zhang, J. Zareno, A. F. Horwitz and C. L. Fraser, ACS Nano, 2008, 2(6), 1252-1258.

7 Z. Zhou, D. Li, H. Yang, Y. Zhu and S. Yang, Dalton Trans., 2011, 40(44), 11941-11944.

8 R. I. Dmitriev, S. M. Borisov, H. Düssmann, S. Sun, B. J. Müller, J. Prehn and D. B. Papkovsky, ACS Nano, 2015, 9(5), 5275-5288.

9 Z. Huang, X. Zhang, X. Zhang, C. Fu, K. Wang, J. Yuan, L. Tao and Y. Wei, Polym. Chem., 2015, 6(4), 607-612.

10 X. Zhang, X. Zhang, B. Yang, J. Hui, M. Liu, Z. Chi, S. Liu, J. Xu and Y. Wie, J. Mater. Chem. C, 2014, 2(5), 816-820.

11 H. Shi, X. Ma, Q. Zhao, B. Liu, Q. Qu, Z. An and W. Huang, Adv. Funct. Mater., 2014, 24(30), 4823-4830.
12 A. Baschieri, F. Monti, N. Armaroli, G. Mazzotti, L. Giorgini, L. Sambri and T. Benelli, Dyes Pigm., 2019, 160, 188-197.

13 A. Baschieri, S. Muzzioli, V. Fiorini, E. Matteucci, M. Massi, L. Sambri and S. Stagni, Organometallics, 2014, 33, 61546164.

14 E. Locatelli and M. Comes Franchini, J. Nanopart. Res., 2012, 14, 1316-1333.

15 M. Comes Franchini, J. Ponti, R. Lemor, M. Fournelle, F. Broggi and E. Locatelli, J. Mater. Chem., 2010, 20(48), 10908-10914.

16 I. Monaco, S. Camorani, D. Colecchia, E. Locatelli, P. Calandro, A. Oudin, S. Niclou, C. Arra, M. Chiariello, L. Cerchia and M. Comes Franchini, J. Med. Chem., 2017, 60(10), 4510-4516.

17 Y. M. Wang, B. Judkewitz, C. A. Di Marzio and C. Yang, Nat. Commun., 2012, 3, 928-936.

18 Z. Starosolski, R. Bhavane, K. B. Ghaghada, S. A. Vasudevan, A. Kaay and A. Annapragada, PLoS One, 2017, 12(11), e0187563.

19 M. Rajamanickam-Vijayakumar, R. Kosuru, S. K. Singh, C. Bhushan Prasad, G. Narayan, M. S. Muthua and S. Singh, RSC Adv., 2016, 6, 74254-74268.

20 M. Naddaka, E. Locatelli, D. Colecchia, L. Sambri, I. Monaco, A. Baschieri, F. Sasdelli, M. Chiariello, E. Matteucci, P. Zani and M. Comes Franchini, RSC Adv., 2015, 5, 1091-1099. 